\title{
On Controllability of Demand Response Resources \& Aggregators' Bidding Strategies
}

\author{
K. Bruninx *†‡, H. Pandžić ${ }^{*}$, H. Le Cadre ${ }^{\ddagger \dagger}$ and E. Delarue ${ }^{* \dagger}$ \\ * Department of Mechanical Engineering, KU Leuven, Leuven, Belgium \\ $\dagger$ EnergyVille, Genk, Belgium \\ $\ddagger$ VITO, Mol, Belgium \\ $\S$ Faculty of Electrical Engineering and Computing, University of Zagreb, Zagreb, Croatia
}

\begin{abstract}
European legislation encourages prosumers to generate, consume and sell self-generated electricity in local and large-scale electricity markets. Since the capacity of residential prosumers is typically insufficient to take part in wholesale markets, aggregators act as mediators. This allows prosumers to monetize the flexibility of their loads through demand response (DR). This paper analyzes the strategic behavior of a DR aggregator, responsible for the optimization of its DR providers' bids in a wholesale market. The DR resource consists of an aggregation of residential electric heating systems, governed by a physical load model and user-specified comfort constraints. The interaction between the aggregator and the DR providers is modelled via Nash Bargaining Theory. Possible deviations from the scheduled heating patterns are accounted for via chance constraints. Results show that limitedly controllable DR resources and a risk-averse aggregator may significantly reduce the monetary benefits of DR.
\end{abstract}

Index Terms-Aggregator, Demand Response, Chanceconstrained Programming, Nash Bargaining Theory

\section{INTRODUCTION}

Residential demand response (DR) resources, such as thermostatically controlled loads (TCLs), may offer the required flexibility to cost-effectively integrate intermittent electricity generation from renewable energy sources (RES) [1]. The whole-system value of these distributed DR resources has been extensively studied in scientific literature (e.g., [2]). This value has been recognized by the European Commission, which encourages member states to open electricity markets for DR resources [3]. System-level studies, e.g. [2], however, do not acknowledge the necessity of an aggregator to bring this small-scale flexibility to large-scale markets. Two distinct perspectives may be adopted to study the interaction between the DR aggregator and an electricity market. First, one may study the aggregator as a non-strategic, price-taking agent via optimization models. For example, Xu et al. [4] study a risk-averse aggregator of distributed generation and electric vehicles. Similarly, Mathieu et al. [5] calculate an upper bound

The work of H. Pandžić was carried out within the projects microGRId Positioning - uGRIP (co-funded by Croatian Environmental Protection and Energy Efficiency Fund through ERA-Net SG+ Programme) and DTP1-5023.2-3Smart (co-funded by European Regional Development Fund through Interreg Danube Transnational Programme). K. Bruninx is a post-doctoral research fellow of the Research Foundation - Flanders (FWO) and the Flemish Institute for Technological Research (VITO). to the profit a price-taking aggregator of TCLs may attain via arbitrage in intraday electricity markets. Alternatively, a bilevel optimization problem may be used to represent the relation between a strategic, price-making agent and a market clearing, reflecting a Stackelberg Game [6], [7]. Kazempour et al. [6] optimize bidding curves for a single large consumer under uncertainty on the offers and demand bids of other nonstrategic agents. Kardakos et al. [7] develop optimal bidding strategies for a virtual power plant consisting of, i.a., DR loads.

Similarly, the interaction between an aggregator and its DR providers may be represented as a Stackelberg game [8], [9]. Li et al. [8] study an aggregator maximizing the social welfare of a group of TCLs, subject to a peak demand constraint, by defining proper retail price signals. Yu and Hong [9] formulate a single leader, multiple follower Stackelberg game to determine optimal control signals for DR loads. Alternatively, several authors have proposed Nash Bargaining theory to mimic the long-term DR provider-aggregator interaction [10]-[12]. Contreras et al. [10] study the repeated interaction between an aggregator, strategically participating in the wholesale market, and distributed, perfectly controllable energy storage systems. Hoa et al. [11] use a Nash Bargaining Game to study the allocation of power/energy to a number of aggregators managing, i.a., TCLs.

In this paper, we study the strategic participation of an aggregator in a wholesale market via a Stackelberg game and the interaction of said aggregator with its DR providers in a Nash Bargaining Game. The limited controllability of the DR resources [13], represented via a physical state-space model as advocated in [14], introduces uncertainty in the aggregator's decision problem and is explicitly considered via chance constraints [2]. This modeling framework allows studying the strategic participation of an aggregator in market while guaranteeing that all user-defined comfort constraints are met with a predefined probability, enforced via the aforementioned chance constraints. A case study inspired on a possible future Belgian power system illustrates the impact of limited controllability and risk aversion on consumer benefits and comfort, aggregator profit and total surplus. To the best of the authors' knowledge, this is the first paper that combines (i) a Stackelberg game between a price-making DR aggregator and a market operator; (ii) chance constraints accounting for the limited controllability of DR loads; and (iii) a Nash Bargaining Game to represent the interaction between the DR providers 


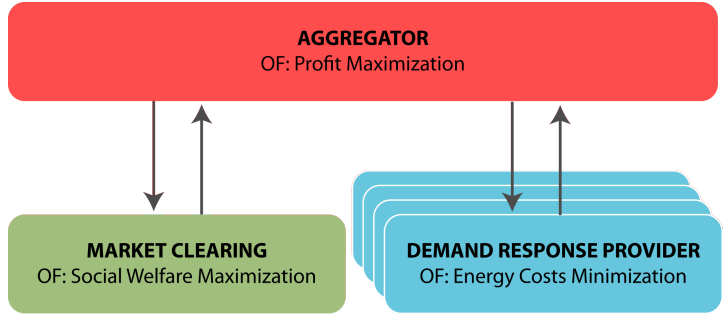

Figure 1. Interaction between the aggregator, the market and the demand response providers and their objective functions (OF).

and the aggregator. The presented methodology constitutes a first step towards a comprehensive framework that may be used by regulators, policy makers and power system operators to assess the value of DR in a deregulated power system or directly integrated in the daily routine of DR aggregators.

The remainder of this paper continues as follows. Section II-A introduces the agents (aggregator, market operator and DR providers), their objectives and the interaction between the agents. The mathematical formulation of the game is presented in Section II-C. A case study is discussed in Section III. Section IV provides some concluding remarks.

\section{Methodology}

First, we provide a brief description of the agents, their objectives and the interactions between the agents. Second, the notation used in this paper is listed. Last, the mathematical formulation of the optimization problem solved by each agent and their dependencies are discussed in detail.

\section{A. Description of the game}

1) Agents: We consider three types of agents: the aggregator, the DR providers and the market operator (Fig. 1). The market operator maximizes the total surplus and ensures the demand for electricity is met by conventional and renewable generation. The DR providers each minimize their energy cost associated with space heating and domestic hot water consumption, which is governed by user-specified comfort constraints. The aggregator attempts to maximize its operating profit. Its revenue depends on the energy consumption of the DR providers and the retail price the aggregator charges. The expenses of the aggregator depend on the wholesale market clearing price.

2) Interactions between agents: The aggregator (leader) decides on its bid in the wholesale market (follower) and the price charged to the DR aggregators (followers) based on its expectation of the wholesale market clearing and the response of its DR consumers. This expectation is governed by a model of the wholesale market clearing and the DR providers. We will leverage chance constrained programming to reflect the limited controllability of DR loads [2]. The interaction between the DR provider may take two distinct forms, which we will refer to as 'retailer' and 'aggregator'. In the retailer-paradigm, the consumers face a flat rate and minimize their own energy use. If the aggregator and the DR providers collaborate to minimize the consumers' energy costs, the game governing the division of the benefits of this interaction may be represented as a Nash Bargaining game.
3) Assumptions: The aggregator is the only strategic pricemaker in the wholesale market. Demand response providers may not switch between DR aggregators or retailers. We only consider the wholesale market, neglecting arbitrage opportunities with intraday and balancing markets. Transmission constraints are not considered in the market clearing problem, as in [2]. Demand response providers may be limitedly controllable. The possible deviations from their expected demand are represented via normal distributions (Eq. (3)).

\section{B. Notation}

\section{1) Sets:}

$\mathcal{H} \quad$ Set of buildings, indexed by $h$.

$\mathcal{I} \quad$ Set of generators, indexed by $i$.

$\mathcal{K} \quad$ Set of non-controllable renewable sources, e.g. wind farms, indexed by $k$.

$\mathcal{P} \quad$ Set of states in the state-space model, indexed by $p$.

$\mathcal{S} \quad$ Set of heat sources, indexed by $s$.

$\mathcal{T} \quad$ Set of time periods, indexed by $t$.

2) Primal variables: ${ }^{1}$

$\alpha_{h, t}^{\mathrm{A}} \quad$ Retail electricity price for consumer $h$ at time period $t, € / \mathrm{MWh}$.

$d_{t} \quad$ Electricity purchased by demand at time period $t$, MWh.

$d_{h, t}^{\mathrm{H}} \quad$ Electricity demand of building $h$ at time period $t$, MWh.

$D_{t}^{\mathrm{H}} \quad$ Stochastic electricity demand of all buildings at time period $t$, MWh.

$g_{i, t} \quad$ Electricity sold by generator $i$ at time period $t$, MWh.

$\Pi \quad$ Aggregator (A) or retailer (R) profit, $€$.

$\psi_{t} \quad$ Auxiliary variable.

$p_{h, t} \quad$ Electric power usage of heat pump (HP) or auxiliary heater $(\mathrm{AH})$ in building $h$ for space heating $(\mathrm{SH})$ or hot water production (HW) at time period $t, \mathrm{MW}$.

$q_{t}^{\text {agg }} \quad$ Electricity purchased by the aggregator at time period $t, \mathrm{MWh}$

$\dot{q}_{h, s, t} \quad$ Heat source $s$ for space heating (SH) or hot water production (HW) in building $h$ at time step $t, \mathrm{MWh}$.

$\overline{Q_{t}^{a g g}} \quad$ Aggregator's bidding quantity at time period $t$, MWh.

$R_{h} \quad$ Aggregator (A) or retailer ( $\mathrm{R}$ ) revenue from building owner $h, €$.

$t_{h, p, t} \quad$ Temperature in state $p$ in building $h$ at time step $t$, K.

$w_{k, t} \quad$ Electricity sold by non-controllable renewable source $k$ at time period $t$, MWh.

\section{3) Parameters:}

$A_{h, p} \quad$ State-space model matrix.

$B_{h, p} \quad$ State-space model matrix, K/MWh.

$C O P_{h} \quad$ Coefficient of performance of heat pump $h$ used for space heating $(\mathrm{SH})$ or hot water production $(\mathrm{HW})$.

$\delta \quad$ Normally distributed disturbance, i.e., $\delta \sim N(0, \sigma)$, proportional (P) or non-proportional (NP).

\footnotetext{
${ }^{1}$ Dual variables are listed after a colon in each constraint and are not listed here. If a variable appears with different superscripts, these superscripts are listed between parentheses in the description of the variable.
} 
$\overline{D_{t}} \quad$ Capacity of demand during time period $t$, MW.

$\epsilon \quad$ Characterization of the aggregator's risk attitude.

$E_{h, p, t} \quad$ Disturbance in state $p(\mathrm{SH})$ or the hot water tank (HW) of building $h$ at time step $t, \mathrm{~K}$.

$\overline{G_{i}} \quad$ Capacity of generator $i$, MW.

$N B_{h} \quad$ Number of buildings of category $h$.

$\Phi^{-1} \quad$ Inverse cumulative probability density function of the standard Normal distribution.

$P^{\text {bid }} \quad$ Aggregator's bidding price, $€ / \mathrm{MWh}$.

$P_{i}^{\mathrm{g}} \quad$ Offering price of generator $i, € / \mathrm{MWh}$.

$P^{\mathrm{d}} \quad$ Bidding price of demand, $€ / \mathrm{MWh}$.

$\overline{P_{h}} \quad$ Capacity of heat pump or auxiliary heater $h$, MW.

$\overline{T_{h, p, t}} \quad$ Upper temperature bound on state $p(\mathrm{SH})$ or hot water tank (HW) of building $h$ at time period $t, \mathrm{~K}$.

$T_{h, p, t} \quad$ Lower temperature bound on state $p(\mathrm{SH})$ or hot water tank (HW) of building $h$ at time period $t, \mathrm{~K}$.

$\overline{W_{k, t}}$ Available output of non-controllable renewable source $k$ at time period $t$, MW.

\section{Mathematical Formulation}

First, we discuss the optimization problem faced by each of the agents (Sections II-C1, II-C2 and II-C3). Second, we describe the interaction between the aggregator and the market operator (Section II-C4) and the aggregator and the DR providers (Section II-C5). Third, the resulting MPECs are reformulated (using KKT conditions, the strong duality theorem and linearization techniques) as equivalent mixed integer quadratically constrained programming (MIQCP) problems in Section II-C6.

1) Aggregator's Perspective: The aggregator aims to maximize its profit, which is the difference between the revenue it obtains from its consumers and the cost of procuring electricity in the wholesale electricity market (Eq. (1)):

$$
\text { Maximize } \Pi^{\mathrm{A}}=\sum_{t \in \mathcal{T}}\left[R^{\mathrm{A}}\left(\alpha_{h, t}^{\mathrm{A}}, D_{t}^{\mathrm{H}}\right)-\alpha_{t} \cdot q_{t}^{\mathrm{agg}}\right]
$$

subject to

$$
\mathbb{P}\left(\overline{Q_{t}^{\mathrm{agg}}} \geq D_{t}^{\mathrm{H}}, \forall t \in \mathcal{T}\right) \geq 1-\epsilon
$$

The aggregrator charges consumers a consumer-specific and time-variant retail rate $\alpha_{h, t}^{\mathrm{A}}$, resulting in revenue $R^{\mathrm{A}}\left(\alpha_{h, t}^{\mathrm{A}}, D_{t}^{\mathrm{H}}\right)$, required to cover its expenses in the wholesale market $\alpha_{t} \cdot q_{t}^{\text {agg }}$. Note that the specific form of the revenue $R^{\mathrm{A}}\left(\alpha_{h, t}^{\mathrm{A}}, D_{t}^{\mathrm{H}}\right)$ depends on the interaction between DR providers and the aggregator (Section II-C5). Likewise, the interaction between the aggregator and the market clearing will determine the wholesale price $\alpha_{t}$, hence the aggregator's expenses (Section II-C4). Chance constraint (2) forces aggregator to procure sufficient electricity to cover the demand of its consumers $D_{t}^{\mathrm{H}}$ at each time step with a probability of $(1-\epsilon) \cdot 100 \%$. In this paper, we assume that this stochastic demand can be characterized by a proportional $\left(\delta^{\mathrm{P}}\right)$ and a nonproportional $\left(\delta^{\mathrm{NP}}\right)$ deviation from an expected demand profile $\sum_{h \in H} N B_{h} \cdot d_{h, t}^{\mathrm{H}}$, governed by Eqs. (12)-(21), as in [2]:

$$
D_{t}^{\mathrm{H}}=\left(1+\delta^{\mathrm{P}}\right) \cdot \sum_{h \in \mathcal{H}} N B_{h} \cdot d_{h, t}^{\mathrm{H}}+\delta^{\mathrm{NP}}, \forall t \in \mathcal{T}
$$

$\delta^{\mathrm{P}}$ and $\delta^{\mathrm{NP}}$ are assumed to follow a Normal distribution, i.e., $\delta^{\mathrm{P}} \sim N\left(0,\left(\sigma^{\mathrm{P}}\right)^{2}\right)$ and $\delta^{\mathrm{NP}} \sim N\left(0,\left(\sigma^{\mathrm{NP}}\right)^{2}\right)$ [2]. Under these assumptions, chance constraint (2) can be analytically recast as the following second order conic (SOC) constraint [2], [15]:

$$
\begin{aligned}
& \overline{Q_{t}^{\mathrm{agg}}}=\sum_{h \in H} N B_{h} \cdot d_{h, t}^{\mathrm{H}}+\Phi^{-1}(1-\epsilon) \cdot\left(\psi_{t}+\sigma^{\mathrm{NP}}\right), \forall t \in \mathcal{T} \\
& \psi_{t}^{2} \geq\left(\sigma^{\mathrm{P}} \cdot \sum_{h \in H} N B_{h} \cdot d_{h, t}^{\mathrm{H}}\right)^{2}, \forall t \in \mathcal{T}
\end{aligned}
$$

where $\psi_{t}$ is an auxiliary decision variable and $\Phi^{-1}$ the inverse cumulative probability density function of the standard Normal distribution. The decision variables of the aggregator are the retail tariff $\alpha_{h, t}^{\mathrm{A}}$ and the demand bid $\overline{Q_{t}^{\mathrm{agg}}}$. The risk-attitude of the aggregator, reflected by parameter $\epsilon$, is assumed to be known.

2) Market Operator's Perspective: The market operator aims to maximize the total surplus with respect to the bids and offers of the market participants. The problem is formulated as follows, based on Pandžić et al. [16] (dual variables of each constraint are listed after a colon):

$$
\text { Maximize } \sum_{t \in \mathcal{T}}\left[P^{\mathrm{d}} \cdot d_{t}+P^{\mathrm{bid}} \cdot q_{t}^{\mathrm{agg}}-\sum_{i \in \mathcal{I}} P_{i}^{\mathrm{g}} \cdot g_{i, t}\right]
$$

subject to:

$$
\begin{gathered}
-\sum_{k \in \mathcal{K}} w_{k, t}-\sum_{i \in \mathcal{I}} g_{i, t}+d_{t}+q_{t}^{\mathrm{agg}}=0, \quad \forall t \in \mathcal{T} \quad: \alpha_{t} \\
0 \leq g_{i, t} \leq \overline{G_{i}}, \quad \forall i \in \mathcal{I}, t \in \mathcal{T} \quad: \quad \underline{\delta_{i, t}}, \overline{\delta_{i, t}} \\
0 \leq d_{t} \leq \overline{D_{t}}, \quad \forall t \in \mathcal{T} \quad: \underline{\epsilon_{t}, \overline{\epsilon_{t}}} \\
0 \leq w_{k, t} \leq \overline{W_{k, t}}, \quad \forall k \in \mathcal{K}, t \in \mathcal{T} \quad: \underline{\zeta_{k, t}}, \overline{\zeta_{k, t}} \\
0 \leq q_{t}^{\mathrm{agg}} \leq \overline{Q_{t}^{\mathrm{agg}}}, \quad \forall t \in \mathcal{T} \quad: \underline{\kappa_{t}}, \overline{\kappa_{t}}
\end{gathered}
$$

Constraint (7) is the power balance equation and includes injections by RES and generators, as well as demand and aggregator quantities. Constraint (8) ensures a generator cannot sell electricity above its capacity, while (9) limits the electricity purchased by the demands. Constraint (10) limits the electricity sold by a non-controllable renewable source to its available generation at time period $t$. The volume of electricity procured by the aggregator, $q_{t}^{\mathrm{agg}}$, is limited to its bid at each time step, $\overline{Q_{t}^{\mathrm{agg}}}$, in Eq. (11).

3) Demand Response Provider's Perspective: Each residential DR provider $h($ set $\mathcal{H})$ minimizes the cost of electric space heating and hot water production while maintaining thermal comfort, leveraging the inherent thermal inertia of the building shell and the hot water tank to shift their electricity consumption $d_{h, t}^{\mathrm{H}}$ to periods of low electricity prices $\alpha_{h, t}^{\mathrm{A}}$ :

$$
\text { Minimize } \sum_{t \in \mathcal{T}} \alpha_{h, t}^{\mathrm{A}} \cdot d_{h, t}^{\mathrm{H}}
$$

subject to

$$
\begin{gathered}
d_{h, t}^{\mathrm{H}}=p_{h, t}^{\mathrm{HP}, \mathrm{SH}}+p_{h, t}^{\mathrm{HP}, \mathrm{HW}}+p_{h, t}^{\mathrm{AH}, \mathrm{SH}}+p_{h, t}^{\mathrm{AH}, \mathrm{HW}}, \forall t \in \mathcal{T} \\
p_{h, t}^{\mathrm{HP}, \mathrm{SH}}+p_{h, t}^{\mathrm{HP}, \mathrm{HW}} \leq \overline{P_{h}^{\mathrm{HP}}}, \forall t \in \mathcal{T} \\
p_{h, t}^{\mathrm{AH}, \mathrm{SH}}+p_{h, t}^{\mathrm{AH}, \mathrm{HW}} \leq \overline{P_{h}^{\mathrm{AH}}}, \forall t \in \mathcal{T} \\
\sum_{s \in \mathcal{S}} \dot{q}_{h, s, t}^{\mathrm{SH}}=C O P_{h}^{\mathrm{SH}} \cdot p_{h, t}^{\mathrm{HP}, \mathrm{SH}}+p_{h, t}^{\mathrm{AH}, \mathrm{SH}}, \forall t \in \mathcal{T}
\end{gathered}
$$

$t_{h, p, t}^{\mathrm{SH}}=A_{h, p}^{\mathrm{SH}} \cdot t_{h, p, t-1}^{\mathrm{SH}}+\sum_{s \in \mathcal{S}} B_{h, p, s}^{\mathrm{SH}} \dot{q}_{\mathrm{h}, \mathrm{s}, \mathrm{t}}^{\mathrm{SH}}+E_{h, p, t}^{\mathrm{SH}}, \forall t \in \mathcal{T}, p \in \mathcal{P}$

$$
\underline{T_{h, p, t}^{\mathrm{SH}}} \leq t_{h, p, t}^{\mathrm{SH}} \leq \overline{T_{h, p, t}^{\mathrm{SH}}}, \forall t \in \mathcal{T}, p \in \mathcal{P}
$$




$$
\begin{gathered}
\dot{q}_{h, t}^{\mathrm{HW}}=C O P_{h}^{\mathrm{HW}} \cdot p_{h, t}^{\mathrm{HP}, \mathrm{HW}}+p_{h, t}^{\mathrm{AH}, \mathrm{HW}}, \forall t \in \mathcal{T} \\
t_{h, t}^{\mathrm{HW}}=A_{h}^{\mathrm{HW}} \cdot t_{h, t-1}^{\mathrm{HW}}+B_{h}^{\mathrm{HW}} \cdot \dot{q}_{\mathrm{h}, \mathrm{t}}^{\mathrm{HW}}+E_{h, t}^{\mathrm{HW}}, \forall t \in \mathcal{T} \\
T_{h, t}^{\mathrm{HW}} \leq t_{h, t}^{\mathrm{HW}} \leq \overline{T_{h}^{\mathrm{HW}}}, \forall t \in \mathcal{T}
\end{gathered}
$$

Each building is equipped with a heat pump (HP) and an auxiliary heater $(\mathrm{AH})$ to provide space heating $(\mathrm{SH})$ and hot water (HW). Equation (13) aggregates the electrical power required by heat pumps $\left(p_{h, t}^{\mathrm{HP}, \mathrm{SH}}, p_{h, t}^{\mathrm{HP}, \mathrm{HW}}\right)$ and auxiliary heaters $\left(p_{h, t}^{\mathrm{AH}, \mathrm{SH}}, p_{h, t}^{\mathrm{AH}, \mathrm{HW}}\right)$, which are limited to their nameplate capacity $\overline{P_{h}^{\mathrm{HP}}}$ and $\overline{P_{h}^{\mathrm{AH}}}$ (Eq. (14)-(15)). We distinguish between the electrical power required for space heating $\left(p_{\mathrm{h}, \mathrm{t}}^{\mathrm{HPH}} \geq 0\right.$ and $\left.p_{h, t}^{\mathrm{AH}, \mathrm{SH}} \geq 0\right)$ and hot water production $\left(p_{h, t}^{\mathrm{HP}, \mathrm{HW}} \geq 0\right.$ and $p_{h, t}^{\mathrm{AH}, \mathrm{HW}} \geq 0$ ). Equations (16) and (19) link the electrical power consumed by the heat pump and the auxiliary heater to the required thermal power for space heating $\dot{q}_{h, s, t}^{\mathrm{SH}} \geq 0$ and hot water production $\dot{q}_{h, t}^{\mathrm{HW}} \geq 0$ using the coefficient of performance $\left(C O P_{h}^{\mathrm{SH}}, C O P_{h}^{\mathrm{HW}}\right)$ of each heat pump. The thermal behavior of each building and its heat emission system is simulated by a linear state-space model $\left(A_{h, p}^{\mathrm{SH}}, B_{h, p}^{\mathrm{SH}}\right)$ (see [14] and references therein). Equation (17) describes the evolution of the temperature $\left(t_{h, p, t}^{\mathrm{SH}} \geq 0\right)$ in each state $p$ (set $\mathcal{P}$ ), which is related to the thermal power supplied to each building $\left(\dot{q}_{h, s, t}^{\mathrm{SH}}\right)$ and the thermal losses, internal and solar gains $E_{h, p, t}^{\mathrm{SH}}$. Equation (18) enforces the user-defined comfort constraints imposed on the indoor air temperature $\left(T_{h, p, t}^{\mathrm{SH}}\right.$, $\overline{T_{h, p, t}^{\mathrm{SH}}}$ ). Similarly, constraint (20) describes the evolution of the temperature of the water $t_{h, t}^{\mathrm{HW}}$ in the hot water storage tank, which depends on the thermal power supplied to the hot water storage tank $\dot{q}_{h, t}^{\mathrm{HW}}$ and the withdrawal of thermal power due to hot water consumption and thermal losses $E_{h, t}^{\mathrm{HW}}$. Equation (21) ensures the user-defined availability of hot water at temperatures between $T_{h, t}^{\mathrm{HW}}$ and $\overline{T_{h, t}^{\mathrm{HW}}}$.

4) Aggregator - Market Interaction: The aggregator acts as a price-maker in the market by bidding strategically, leveraging the flexibility of its consumers to minimize its procurement cost. Given this relation and neglecting the interaction with DR providers, the problem can be represented as a bilevel optimization problem:

\section{Maximize (1)}

subject to

Chance constraints: (4) - (5)

Market clearing: Maximize (6) s.t. (7) - (11)

The MPEC equivalent of the bilevel model above is obtained by replacing the lower-level market clearing objective (6) by the KKT conditions associated with the market clearing problem (Eqs. (6)-(11)):

\section{Stationarity conditions:}

$$
\begin{gathered}
P^{\mathrm{d}}-\alpha_{t}-\overline{\epsilon_{t}}+\underline{\epsilon_{t}}=0, \quad \forall t \in \mathcal{T} \\
P^{\mathrm{bid}}-\alpha_{t}-\overline{\kappa_{t}}+\underline{\kappa_{t}}=0, \quad \forall t \in \mathcal{T} \\
-P_{i}^{\mathrm{g}}+\alpha_{t}-\overline{\delta_{i, t}}+\underline{\delta_{i, t}}=0, \quad \forall i \in \mathcal{I}, t \in \mathcal{T} \\
\alpha_{t}-\overline{\zeta_{k, t}}+\underline{\zeta_{k, t}}=0, \quad \forall k \in \mathcal{K}, t \in \mathcal{T}
\end{gathered}
$$

Complementary slackness conditions:

$$
\begin{gathered}
0 \leq g_{i, t} \perp \overline{\delta_{i, t}} \geq 0, \quad \forall i \in \mathcal{I}, t \in \mathcal{T} \\
0 \leq\left(\overline{G_{i}}-g_{i, t}\right) \perp \overline{\delta_{i, t}} \geq 0, \quad \forall i \in \mathcal{I}, t \in \mathcal{T} \\
0 \leq d_{t} \perp \overline{\epsilon_{t}} \geq 0, \quad \forall t \in \mathcal{T} \\
0 \leq\left(\overline{D_{t}}-d_{t}\right) \perp \overline{\epsilon_{t}} \geq 0, \quad \forall t \in \mathcal{T} \\
0 \leq w_{k, t} \perp \underline{\zeta_{k, t}} \geq 0, \quad \forall k \in \mathcal{K}, t \in \mathcal{T} \\
0 \leq\left(\overline{W_{k, t}}-w_{k, t}\right) \perp \overline{\zeta_{k, t}} \geq 0, \quad \forall k \in \mathcal{K}, t \in \mathcal{T} \\
0 \leq q_{t}^{\text {agg }} \perp \underline{\kappa_{t}} \geq 0, \quad \forall t \in \mathcal{T} \\
0 \leq\left(\overline{Q_{t}^{\text {agg }}}-q_{t}^{\text {agg }}\right) \perp \overline{\kappa_{t}} \geq 0, \quad \forall t \in \mathcal{T}
\end{gathered}
$$

5) Aggregator - Demand Response Provider Interaction: We distinguish between two options for the interaction between the aggregator and the DR provider:

a) Retailer: The aggregator acts as a retailer and offers consumers a flat retail rate $\left(\alpha_{h, t}^{\mathrm{A}}=\alpha^{\mathrm{A}}\right)$, which leads to a complete decoupling between the retailer's procurement problem, as described in Section II-C4, and the consumers problem (Eqs. (12)-(21)). Consumers minimize their energy demand $d_{h, t}^{H}$, which is considered a parameter in the retailer's problem. As a reference case, we assume that the retailer does not make any profit, i.e., the consumers are charged a retail rate that allows the retailer to recover its costs:

$$
R^{\mathrm{A}}\left(\alpha^{\mathrm{A}}, D_{t}^{\mathrm{H}}\right)=\sum_{t \in \mathcal{T}} \alpha_{t} \cdot q_{t}^{\mathrm{agg}}
$$

b) Nash Bargaining: In this paradigm, DR providers collaborate with the aggregator. A DR provider will sign a contract with an aggregator if this reduces its current electricity bill $R_{h}^{\mathrm{R}}$, i.e., if $\sum_{t \in \mathcal{T}} \alpha_{h, t}^{\mathrm{A}} \cdot d_{h, t}^{\mathrm{H}} \leq R_{h}^{\mathrm{R}}$. Similarly, an aggregator will offer a rate $\alpha_{h, t}^{\mathrm{A}}$ to a DR provider if this results in a profit, i.e., if $\sum_{h \in \mathcal{H}} N B_{h} \cdot \sum_{t \in \mathcal{T}} \alpha_{h, t}^{\mathrm{A}} \cdot d_{h, t}^{\mathrm{H}} \geq \sum_{t \in \mathcal{T}} \alpha_{t} \cdot q_{t}^{\mathrm{agg}}$. If a rate $\alpha_{h, t}^{\mathrm{A}}$ exists in which the previous conditions hold, this tariff may be the result of a Nash Bargaining Game [17]. The overall monetary benefits of the collaboration between the aggregator and the DR providers $\mathcal{B}$ can be calculated as $\sum_{h \in \mathcal{H}} R_{h}^{\mathrm{R}}-\sum_{t \in \mathcal{T}} \alpha_{t} \cdot q_{t}^{\mathrm{agg}}$. The Bargaining Game determines how the benefits of this collaboration are shared between the DR providers and the aggregator, according to division factors $x_{h}^{\mathrm{H}}$ and $x^{\mathrm{A}}\left(\sum_{h \in \mathcal{H}} x_{h}^{\mathrm{H}}+x^{\mathrm{A}}=1, x_{h}^{\mathrm{H}}, x^{\mathrm{A}} \in[0,1]\right)$. In contrast, if the aggregator and the DR provider do not reach an agreement, the aggregator receives no profit from that consumers and the consumer's electricity bill remains unchanged. Under certain assumptions [17], this game can formally be described as the following optimization problem, with $y_{h}^{\mathrm{H}}$ and $y^{\mathrm{A}}$ the exogenous bargaining power of consumer $h$ and the aggregator $\left(y_{h}^{\mathrm{H}}, y^{\mathrm{A}} \in[0,1], \sum_{h \in \mathcal{H}} y_{h}^{\mathrm{H}}+y^{\mathrm{A}}=1\right)$ :

$$
\text { Maximize } \prod_{h \in \mathcal{H}}\left[x_{h}^{\mathrm{H}} \cdot \mathcal{B}\right]^{y_{h}^{\mathrm{H}}} \cdot\left[x^{\mathrm{A}} \cdot \mathcal{B}\right]^{y^{\mathrm{A}}}
$$

subject to

$$
\begin{aligned}
& \mathcal{B}=\sum_{h \in \mathcal{H}} R_{h}^{\mathrm{R}}-\sum_{t \in \mathcal{T}} \alpha_{t} \cdot q_{t}^{\mathrm{agg}} \\
& \sum_{t \in \mathcal{T}} \alpha_{t}^{\mathrm{A}} \cdot d_{h, t}^{\mathrm{H}}=x_{h}^{\mathrm{H}} \cdot\left(\sum_{h \in \mathcal{H}} R_{h}^{\mathrm{R}}-\sum_{t \in \mathcal{T}} \alpha_{t} \cdot q_{t}^{\mathrm{agg}}\right), \quad \forall h \in \mathcal{H} \\
& \sum_{t \in \mathcal{T}} \alpha_{t}^{\mathrm{A}} \cdot d_{h, t}^{\mathrm{H}} \leq R_{h}^{\mathrm{R}}, \quad \forall h \in \mathcal{H}
\end{aligned}
$$




$$
\sum_{h \in \mathcal{H}} N B_{h} \cdot \sum_{t \in \mathcal{T}} \alpha_{t}^{\mathrm{A}} \cdot d_{h, t}^{\mathrm{H}} \geq \sum_{t \in \mathcal{T}} \alpha_{t} \cdot q_{t}^{\mathrm{agg}}
$$

Aggregator constraints: (4) - (5)

Demand response constraints: (13) - (21)

Market clearing: (7) - (11), (22) - (33)

Assuming the aggregator treats all consumers nondiscriminatory $\left(x_{h}^{\mathrm{H}}=x^{\mathrm{H}}\right)$ and equal bargaining power for the $N$ DR providers $\left(y_{h}^{\mathrm{H}}=y^{\mathrm{H}}\right)$, objective (35) can be recasted as:

$$
\text { Maximize }\left(x^{H}\right)^{y^{H} \cdot N} \cdot\left(1-x^{H} \cdot N\right)^{1-y^{H} \cdot N} \cdot \mathcal{B}
$$

In this paper, we assume the bargaining process on the formation of retail rates, i.e., the division factors $x_{h}^{\mathrm{H}}$ and $x^{\mathrm{A}}$, governing the split of the overall benefit of DR, takes place well before the aggregator offers demand in the wholesale market. Hence, the only term the aggregator can optimize in objective (40) on a day-to-day basis is $\sum_{t \in T} \alpha_{t} \cdot q_{t}^{\text {agg }}$. Note that the outcome of the bargaining game above determines some restrictions on how the aggregator forms retail prices, but does not prescribe the (time-varying) magnitude of the retail tariff. In addition to the distribution of the benefits of DR, the tariff may be used for other purposes, e.g., as a control signal for the DR loads.

\section{6) Equivalent MIQCP formulations:}

a) Retailer: The retailer minimizes its expenses in the wholesale market and determines a flat retail rate $\alpha^{\mathrm{A}}$ to ensure profit-neutrality (34):

subject to

$$
\text { Maximize }-\sum_{t \in \mathcal{T}} \alpha_{t} \cdot q_{t}^{\mathrm{agg}}
$$

Aggregator constraints: (4) - (5), (34)

Market clearing: (7) - (11), (22) - (33)

Recall that $d_{h, t}^{\mathrm{H}}$ is a parameter in the retailer's problem, obtained from solving the DR provider problems (Eqs. (12)(21)) separately, assuming a flat retail rate $\alpha^{\mathrm{A}}$.

b) Nash Bargaining: In the Nash Bargaining paradigm, the aggregator's optimization problem is formulated as:

$$
\text { Maximize }-\sum_{t \in \mathcal{T}} \alpha_{t} \cdot q_{t}^{\mathrm{agg}}
$$

subject to

Aggregator constraints: (4) - (5), (37) - (39)

Demand response constraints: (13) - (21)

Market clearing: (7) $-(11),(22)-(33)$

Each MPEC above contains non-linearities (i) in the objective of the aggregator/retailer and (ii) the complementary slackness conditions (Eqs. (26)-(33)). Via stationarity condition (23) and complementary slackness conditions (32)-(33), we recast the non-linear term $\alpha_{t} \cdot q_{t}^{\text {agg }}$ in the aggregator's objective as:

$$
\sum_{t \in \mathcal{T}} \alpha_{t} \cdot q_{t}^{\mathrm{agg}}=\sum_{t \in \mathcal{T}}\left[P^{\mathrm{bid}} \cdot q_{t}^{\mathrm{agg}}-\overline{Q_{t}^{\mathrm{agg}}} \cdot \overline{\kappa_{t}}\right]
$$

The last term in the equation above is non-linear, but can be expressed as a sum of linear terms via the strong duality equality associated with the dual of the market clearing

$$
\begin{aligned}
\sum_{t \in \mathcal{T}} \overline{\kappa_{t}} \cdot \overline{Q_{t}^{\mathrm{agg}}}= & \sum_{t \in \mathcal{T}}\left(P^{\mathrm{d}} \cdot d_{t}+P^{\mathrm{bid}} \cdot q_{t}^{\mathrm{agg}}-\sum_{i \in \mathcal{I}} P_{i}^{\mathrm{g}} \cdot g_{i, t}\right. \\
& \left.-\sum_{i \in \mathcal{I}} \overline{G_{i}} \cdot \overline{\delta_{i, t}}-\overline{D_{t}} \cdot \overline{\epsilon_{t}}-\sum_{k \in \mathcal{K}} \overline{W_{k, t}} \cdot \overline{\zeta_{k, t}}\right)
\end{aligned}
$$

The non-linearities in the complementary slackness conditions (Eqs. (26)-(33)) can be linearized using the method of Fortuny-Amat and McCarl [18]. With the reformulation of the chance constraint and these linearizations, one obtains two MIQCP problems that can be solved with existing solvers [15].

\section{CASe Study}

We study DR with residential electric heating systems using a model inspired by the current Belgian power system (annual electricity demand: $87 \mathrm{TWh}$, peak demand: $13.8 \mathrm{GW}$ (2017) [19]), documented in [2]. Wind energy is assumed to cover $50 \%$ of the annual energy demand prior to the introduction of electric space heating. Seven GW of gas-fired (six GW CCGTs, one GW OCGTs) and one GW of oil-fired generation is added to the system to cover the additional heating demand. The transmission grid and interconnections with neighboring countries are neglected. The number of buildings is set to $\sum_{h \in \mathcal{H}} N B_{h}=10^{6}$ and the building portfolio is represented by an average 2030 low-energy building described in [2]. The temperature bounds $\left(T_{h, p, t}^{\mathrm{SH}}, T_{h, p, t}^{\mathrm{SH}}, T_{h, t}^{\mathrm{HW}}\right)$ aim to represent possible occupant behavior (see [2] and references therein). Conventional generation is assumed to offer all its capacity at average generation cost. The aggregator and conventional demand bid at the price cap (here: $1000 € / \mathrm{MWh}$ ) to ensure the demand bids are accepted.

All models are implemented in GAMS v24.9 and solved using Gurobi v7.5. The stopping tolerance was set to $1 \%$. Simulations are performed on a $2.8 \mathrm{GHz}$ machine with 8 cores and $8 \mathrm{~GB}$ of RAM. Solving the retailer's problem requires at most 4.2 seconds, compared to up to 1622 seconds (median: 88 seconds) for the aggregator's problem.

In Section III-A, we show how a retailer and an aggregator participate in wholesale markets on the fifth day of week 7 with perfectly and imperfectly controllable DR loads. We focus on price formation, changes in heating demand and the impact of these decisions on total surplus and aggregator profit. Subsequently, we provide a quantitative analysis of the obtained results for week 7 of the calender year with respect to the assumptions on the limited controllability and the risk attitude of the aggregator or retailer. Week 7 was selected as the week showing the highest potential for operating cost reductions based on a simplified analysis, as in [2]. Last, we discuss some limitations of the presented framework.

\section{A. Optimal bidding strategies}

On the fifth day of week 7 , low demand periods coincide with abundant wind power output during the first hours of the day, resulting in nuclear units as the marginal generation resource (Fig. 2a). Under the retailer paradigm, consumers minimize their own energy use, which results in a strong peak in the heating demand in the morning (Fig. 2b). This 


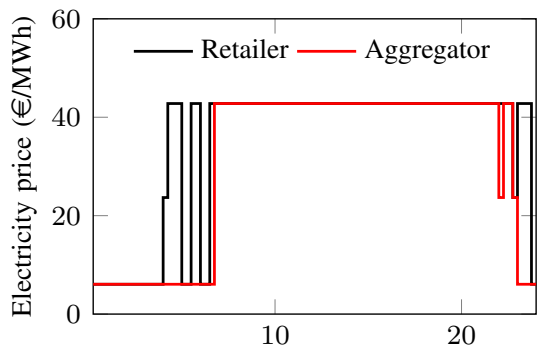

Time (h)

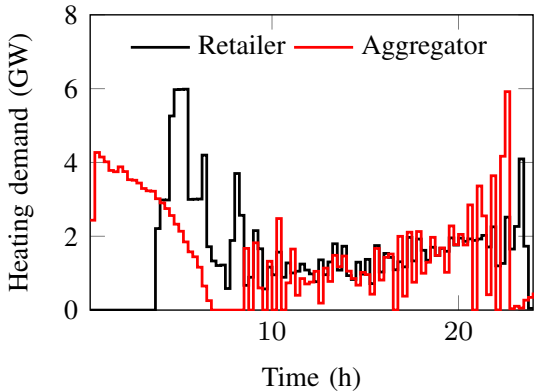

(b) Heating demand

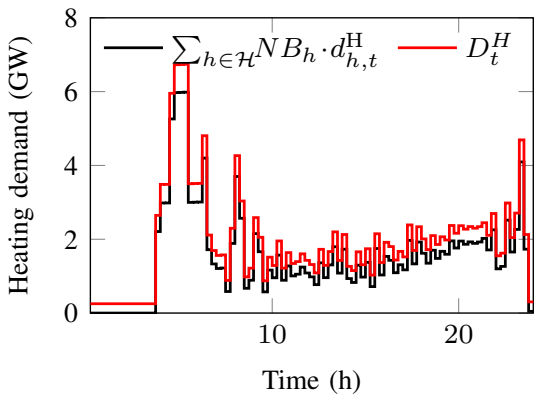

(e) Heating demand (retailer)

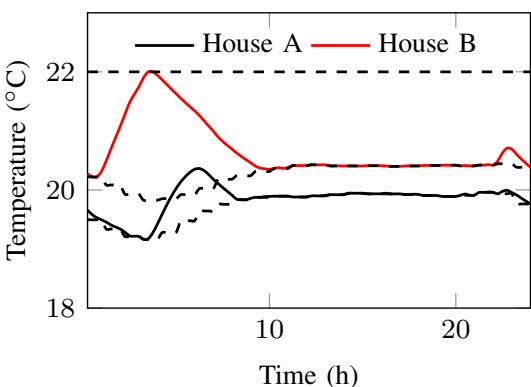

(c) Day-zone temperatures (aggregator)

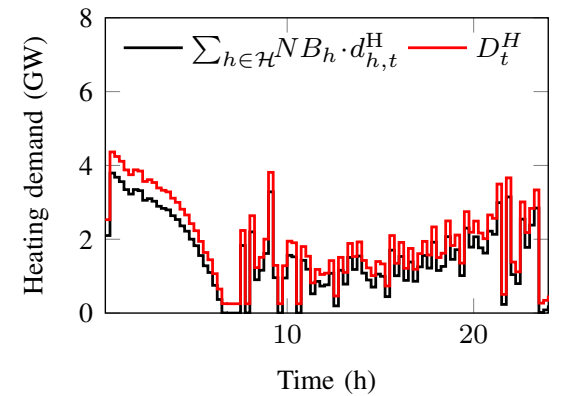

(f) Heating demand (aggregator)

(d) Electricity prices

Figure 2. The market clearing price (left), heating demand (center and right) and the expected day-zone temperatures under the aggregator paradigm (top row, right). In the top row, the DR loads are assumed to be perfectly controllable. In the bottom row, the DR loads control error is assumed to be characterized by $\delta^{\mathrm{NP}} \sim N(0,100 \mathrm{MW})$ and $\delta^{\mathrm{P}} \sim N(0,0.1)$. Parameter $\epsilon$ is equal to 0.2 .

in turn triggers price peaks in the wholesale market (Fig. 2a). Although the heating demand is significantly lower during the rest of the day, the wholesale price remains around $42 € / \mathrm{MWh}$ due to a decreased availability of RES-based generation.

If the aggregator is allowed to shift the heating demand within the user-specified comfort constraints, the morning price peaks are mitigated and the high-price period is shortened (Fig. 2a). This is the result of shifting the heating demand to the earlier hours (Fig. 2b), which results in significant preheating of the considered dwellings (Fig. 2c). On this particular day, this leads to an overall benefit of $2.19 \mathrm{M} €$, which is to be shared by the aggregator and the DR providers.

When the DR loads exhibit limited controllability (or when the heating demand forecast by the retailer is imperfect), the retailer/aggregator must procure more than the expected heating demand $\sum_{h \in \mathcal{H}} N B_{h} \cdot d_{h, t}^{\mathrm{H}}$ (red vs. black line, Fig. 2e) to deal with unexpected real-time deviations from the expected heating demand (black line) ${ }^{2}$. As a result, the high price period spans more hours (Fig. 2d). Leveraging the flexibility of the DR loads allows the aggregator to limit the duration of the high price period (Fig. 2d) by shifting demand to the night (Fig. $2 \mathrm{f}$ ), effectively managing the uncertainty more cost-effectively. The overall cost savings, compared to a setting in which the retailer faces the same controllability issues and exhibits the same risk attitude, amounts to $2.41 \mathrm{M} €$. Compared to a retailer with a perfectly controllable heating load, overall cost savings are limited to $0.67 \mathrm{M} €$.

\footnotetext{
${ }^{2}$ Note that the aggregator may face an excess or deficit of electricity in real time, depending on it's risk attitude $\epsilon$ and the real-time DR load. How the aggregator manages this excess or deficit is, however, out of the scope of this paper (see Section III-C).
}

\section{B. Sensitivity analysis w.r.t. $\epsilon, \sigma^{P}$ and $\sigma^{N P}$}

To study the impact of different $\epsilon, \sigma^{P}$ and $\sigma^{N P}$-values, we repeat the analysis above for the seventh week of the calender year. In Fig. 3, we visualize the change in total surplus, generation costs, overall benefits of the introduction of DR and heating demand for three degrees of limited controllability $\left(\sigma^{N P}=50 \mathrm{MW}, \sigma^{P}=0.05, \sigma^{N P}=100 \mathrm{MW}, \sigma^{P}=0.1, \sigma^{N P}=\right.$ $\left.150 \mathrm{MW}, \sigma^{P}=0.15\right)$. To calculate these changes, we use the results obtained for a retailer with an equivalent, perfectly predictable and controllable heating load as a reference.

As the degree of limited controllability increases (increasing $\sigma$-values) and the aggregator becomes more risk-averse (decreasing $\epsilon$-values), the total surplus in the electricity market increases (Fig. 3a), as the aggregator must procure more energy to meet unexpected real-time increases in demand (Fig. 3b). Consequently, generation costs increase (Fig. 3a) and the overall benefit of the DR provider-aggregator collaboration $\mathcal{B}$ reduces (Fig. 3b). For small $\sigma$-values, overall benefit $\mathcal{B}$ remains positive. At high $\sigma$-values, a risk-averse aggregator may face negative benefits $\mathcal{B}$.

Mathematically, these trends can be explained as follows. Increasing $\sigma$ and decreasing $\epsilon$-values leads to higher aggregator bids ( $Q_{t}^{\text {agg }}$, Eqs. (4)-(5)). This yields a higher total surplus in the market and higher generation costs (Eqs. (6) and (11)). The market operator will accept higher aggregator bids, hence the overall benefit $\mathcal{B}$ and the aggregator's profit decrease.

\section{Limitations of the proposed framework}

Although the presented framework allows for a first analysis of the market participation of an aggregator of limitedly controllable DR loads, several limitations of the proposed 


$$
\begin{aligned}
& \multimap \sigma^{N P}=50 \mathrm{MW}, \sigma^{P}=0.05 \multimap \sigma^{N P}=100 \mathrm{MW}, \sigma^{P}=0.1 \\
& \bullet \sigma^{N P}=150 \mathrm{MW}, \sigma^{P}=0.15
\end{aligned}
$$

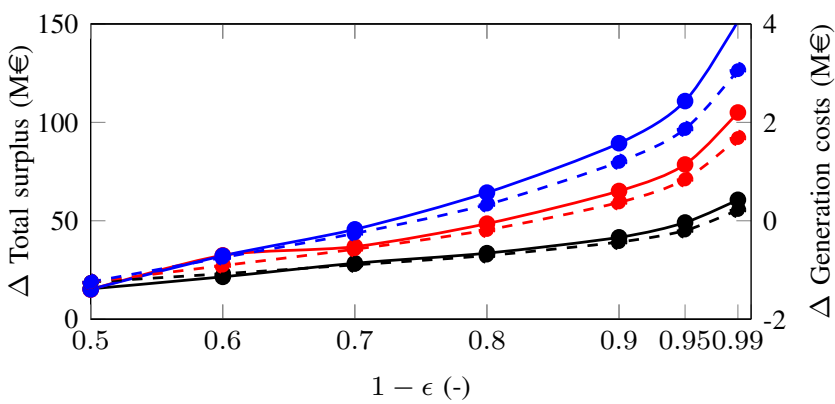

(a) Change in total surplus (solid lines, left axis) and generation costs (dashed lines, right axis).

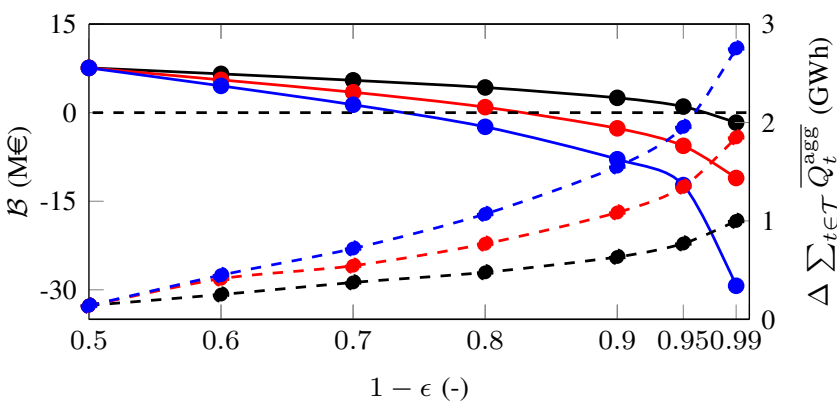

(b) Overall benefit $\mathcal{B}$ (solid lines, left axis) and change in demand procured by the aggregator (dashed lines, right axis).

Figure 3. As the DR load becomes less controllable (higher $\sigma$-values) and the aggregator becomes more risk-averse (lower $\epsilon$-values), the total surplus and generation cost increase (Fig. 3a) and overall benefit $\mathcal{B}$ decrease due to higher demand bids of the aggregator (Fig. 3b).

framework may be identified. First, the proposed method may yield load and dispatch schedules that are infeasible w.r.t. the technical constraints of the transmission and distribution system and/or generation assets. Second, the aggregator has a deterministic anticipation of the market outcome, which does not reflect reality. Third, only the day-ahead market is considered. The aggregator therefore does not has the option to procure/sell deficits/excess energy in the intra-day market. Last, ex-post Monte-Carlo evaluations of the impact of realtime deviations of the scheduled DR loads on the aggregators profit are out of the scope of this paper. Risk-neutral behavior therefore always appears optimal (Fig. 3) and, consequently, one is unable to determine the optimal risk-attitude of the aggregator or to test the robustness of the obtained solution.

\section{CONCLUSION}

Demand response (DR) resources, such as thermostatically controlled loads, may allow compensating for variations and unexpected deviations in electricity generated from renewable energy sources. Aggregators may be needed to bring these distributed, small-scale resources to large-scale markets.

In this paper, we study the interaction between (i) an aggregator and a wholesale market via a Stackelberg game and (ii) an aggregator and its DR providers via a Nash Bargaining Game. To manage the limited controllability of DR loads, we propose chance constraints.

In a case study, inspired by the Belgian power system, we illustrate how the aggregator may lower wholesale prices and how it may manage limitedly controllable resources. As the DR resource becomes less controllable and the aggregator becomes more risk-averse, the overall benefit of DR decreases, whereas the total surplus and generation costs increase.

The presented research may be strengthened by incorporating uncertainty in the anticipation of the aggregator on the market clearing outcome and a validation of this outcome w.r.t. the technical constraints of generation and grid assets. In addition, ex-post Monte Carlo evaluations of the aggregator profit for different realizations of the DR load could allow identifying an optimal risk attitude for the aggregator. Last, introducing multiple segments of consumers, each with a specific bargaining power, could lead to interesting insights.

\section{REFERENCES}

[1] G. Strbac, "Demand side management: Benefits and challenges," Energy Policy, vol. 36, no. 12, pp. 4419-4426, 2008.

[2] K. Bruninx, Y. Dvorkin, E. Delarue, W. D'haeseleer, and D. S. Kirschen, "Valuing demand response controllability via chance constrained programming," IEEE Trans. Sustain. Energy, vol. 9, no. 1, pp. 178-187, 2018.

[3] P. Bertoldi, P. Zancanella, and B. Boza-Kiss, "Demand Response status in EU Member States," EUR 27998 EN, JRC, Tech. Rep., 2016.

[4] Z. Xu, Z. Hu, Y. Song, and J. Wang, "Risk-Averse Optimal Bidding Strategy for Demand-Side Resource Aggregators in Day-Ahead Electricity Markets under Uncertainty," IEEE Trans. Smart Grid, vol. 8, no. 1, pp. 96-105, 2017.

[5] J. L. Mathieu, M. Kamgarpour, J. Lygeros, G. Andersson, and D. S. Callaway, "Arbitraging intraday wholesale energy market prices with aggregations of thermostatic loads," IEEE Trans. Power Syst., vol. 30, no. 2, pp. 763-772, 2015

[6] S. J. Kazempour, A. J. Conejo, and C. Ruiz, "Strategic bidding for a large consumer," IEEE Trans. Power Syst., vol. 30, no. 2, pp. 848-856, 2015.

[7] E. G. Kardakos, C. K. Simoglou, and A. G. Bakirtzis, "Optimal Offering Strategy of a Virtual Power Plant: A Stochastic Bi-Level Approach," IEEE Trans. Smart Grid, vol. 7, no. 2, pp. 794-806, 2016.

[8] S. Li, W. Zhang, J. Lian, and K. Kalsi, "Market-Based Coordination of Thermostatically Controlled Loads - Part II: Unknown Parameters and Case Studies," IEEE Trans. Power Syst., vol. 31, no. 2, pp. 1179-1187, 2016.

[9] M. Yu and S. H. Hong, "A Real-Time Demand-Response Algorithm for Smart Grids: A Stackelberg Game Approach," IEEE Trans. Smart Grid, vol. 7, no. 2, pp. 879-888, 2016

[10] J. E. Contreras-Ocana, M. A. Ortega-Vazquez, and B. Zhang, "Participation of an Energy Storage Aggregator in Electricity Markets," IEEE Trans. Smart Grid, vol. PP, no. 99, pp. 1-14, 2017.

[11] H. Hao, A. Somani, J. Lian, and T. E. Carroll, "Generalized aggregation and coordination of residential loads in a smart community," SmartGridComm 2015, Miama, FL, USA, November 2-5, 2015.

[12] G. Ye, G. Li, D. Wu, X. Chen, and Y. Zhou, "Towards Cost Minimization with Renewable Energy Sharing in Cooperative Residential Communities," IEEE Access, vol. 5, pp. 11688-11 699, 2017.

[13] J. L. Mathieu, M. G. Vaya, and G. Andersson, "Uncertainty in the flexibility of aggregations of demand response resources," IECON 2013 , Vienna, Austria, November 10-13, 2013.

[14] D. Patteeuw, K. Bruninx, A. Arteconi, E. Delarue, W. D'haeseleer, and L. Helsen, "Integrated modeling of active demand response with electric heating systems coupled to thermal energy storage systems," Applied Energy, vol. 151, pp. 306-319, 2015.

[15] D. Bienstock, M. Chertkov, and S. Harnett, "Chance-Constrained Optimal Power Flow: Risk-Aware Network Control under Uncertainty," SIAM Review, vol. 56, no. 3, pp. 461-495, 2014.

[16] H. Pandžić, A. J. Conejo, and I. Kuzle, "An epec approach to the yearly maintenance scheduling of generating units," IEEE Trans. Power Syst., vol. 28, no. 2, pp. 922-930, 2013.

[17] M. J. Osborne and A. Rubinstein, Bargaining and Markets. New York, NY, USA: Academic Press, 1990.

[18] J. Fortuny-Amat and B. McCarl, "A representation and economic interpretation of a two-level programming problem," JORS, vol. 32, no. 9, pp. 783-792, 1981

[19] Elia N.V., "Grid data," Available online: www.elia.be/en/grid-data, 2018. 